\title{
Detection of respiratory viruses by real-time polymerase chain reaction in outpatients with acute respiratory infection
}

\author{
Ronaldo Bragança Martins Júnior ${ }^{1 /+}$, Sharon Carney², Daniel Goldemberg ${ }^{2}$, Lucas Bonine ${ }^{3}$, \\ Liliana Cruz Spano', Marilda Siqueira' ${ }^{2}$, Rita Elizabeth Checon ${ }^{1}$ \\ ${ }^{1}$ Laboratório de Vírus Respiratórios, Núcleo de Doenças Infecciosas, Universidade Federal do Espírito Santo, Vitória, ES, Brasil \\ ${ }^{2}$ Laboratório de Vírus Respiratórios, Instituto Oswaldo Cruz-Fiocruz, Rio de Janeiro, RJ, Brasil \\ ${ }^{3}$ Laboratório Central de Saúde Pública do Espírito Santo, Vitória, ES, Brasil
}

\begin{abstract}
Viruses are the major contributors to the morbidity and mortality of upper and lower acute respiratory infections (ARIS) for all age groups. The aim of this study was to determine the frequencies for a large range of respiratory viruses using a sensitive molecular detection technique in specimens from outpatients of all ages with ARIs. Nasopharyngeal aspirates were obtained from 162 individuals between August 2007-August 2009. Twenty-three pathogenic respiratory agents, 18 respiratory viruses and five bacteria were investigated using multiplex real-time reverse transcriptase polymerase chain reaction (RT-PCR) and indirect immunofluorescence assay (IIF). Through $I I F, 33(20.4 \%)$ specimens with respiratory virus were recognised, with influenza virus representing over half of the positive samples. Through a multiplex real-time RT-PCR assay, 88 (54.3\%) positive samples were detected; the most prevalent respiratory viral pathogens were influenza, human rhinovirus and respiratory syncytial virus (RSV). Six cases of viral co-detection were observed, mainly involving RSV. The use of multiplex real-time RT-PCR increased the viral detection by $33.9 \%$ and revealed a larger number of respiratory viruses implicated in ARI cases, including the most recently described respiratory viruses [human bocavirus, human metapneumovirus, influenza A (HINI) pdm09 virus, human coronavirus (HCoV) NL63 and HCoV HKU1].
\end{abstract}

Key words: respiratory infections - real-time PCR - multiplex - viruses

Acute respiratory infections (ARIs) are the main causes of morbidity and mortality worldwide, especially in children during the first years of life (Osterhaus 2008).

The viruses most commonly associated with ARIs are influenza viruses $A$ and $B(\operatorname{Inf} A$ and $\operatorname{Inf} B)$, respiratory syncytial virus (RSV), parainfluenza viruses 1-4 (PIV 1- 4), human adenovirus (HAdV), human rhinovirus (HRV), human coronavirus ( $\mathrm{HCoV})$ and enterovirus (EV) (Arruda et al. 2006). Since 2001, new viruses have been detected in ARI cases, such as human metapneumovirus (hMPV) (van den Hoogen et al. 2001); two new types of HCoV, HKU1 and NL63 (van der Hoek et al. 2004, Woo et al. 2005) and human bocavirus (HBoV) (Allander et al. 2005).

The diagnosis of respiratory viruses based on virus isolation, detection of antigens or serology is too time-consuming and, in some cases, has low sensitivity (Gunson et al. 2005). Therefore, molecular diagnostic methods using "in-house" or commercially available techniques are alternatives to obtain faster results and higher sensitivity and specificity. These molecular methods can potentially reduce the length of hospitalisa-

doi: 10.1590/0074-0276140046

Financial support: FAPES, FACITEC-Vitória-ES, DECIT-MS, Brasil +Corresponding author: rb.mj@hotmail.com

Received 7 February 2014

Accepted 31 July 2014 tion and unnecessary treatment costs; furthermore, they can also contribute to nosocomial infection control programs and can help to guide therapy (Barenfanger et al. 2000, Mahony 2008, Brittain-Long et al. 2010).

The aim of the present study was to determine the frequencies of a range of respiratory pathogens using the Fast-Track Diagnostics Respiratory Pathogens 21 PLUS (FTDRP 21 plus) multiplex reverse transcriptase polymerase chain reaction (RT-PCR) assay in patients of all ages with ARIs treated in emergency rooms or primary care units.

\section{SUBJECTS, MATERIALS AND METHODS}

This descriptive study was developed in Vitória, Southeast Brazil, from a set of biological samples obtained over a two-year period (August 2007-August 2009) as a part of the Respiratory Virus Surveillance Program of the Ministry of Health, Brazil. Nasopharyngeal aspirates (NPAs) were collected from patients of all ages who were attended at either emergency rooms or primary care units. The patients presented with fever (temperature equal to or greater than $38^{\circ} \mathrm{C}$ ) and runny nose, in addition to one or more of the following symptoms: cough, myalgia, nasal congestion, headache, sore throat and earache, within five days of the symptom onset. The nasopharyngeal aspiration procedure is a part of the routine of the Respiratory Virus Surveillance Program.

All samples were tested by indirect immunofluorescence assay (IIF) and FTDRP 21 plus multiplex realtime RT-PCR assay.

Seven respiratory viruses (RSV, PIV 1-3, Inf A, Inf B and $\mathrm{HAdV}$ ) were screened in all specimens by IIF using 
the Respiratory Panel 1 Viral Screening \& Identification $\mathrm{Kit}^{\mathrm{TM}}$ (Chemicon International, Millipore, USA) according to the manufacturer's instructions.

Twenty-three respiratory pathogens, including 18 human respiratory viruses and five bacterial species, were tested using the multiplex protocol. Total nucleic acid was extracted using the MagNA Pure LC automated extraction system (Roche, Switzerland). Nucleic acid was extracted from $200 \mu \mathrm{L}$ of each NPA sample and was eluted to a final volume of $100 \mu \mathrm{L}$ following the MagNA Pure LC Total Nucleic Acid Isolation v.12.0 (Roche) protocol. An internal RNA virus control, Brome Mosaic Virus (Fast-track Diagnostics, Luxembourg), was introduced into the lysis buffer for each specimen to monitor the sample extraction and reverse transcription. Multiplex real-time RT-PCR was performed using the FTDRP 21 plus according to the manufacturer's instructions (Fast-track Diagnostics). The reaction volume for each test was $25 \mu \mathrm{L}$, made up of $10 \mu \mathrm{L}$ of nucleic acid and 15 $\mu \mathrm{L}$ of buffer/enzyme mix from the AgPath-ID ${ }^{\mathrm{TM}}$ OneStep RT-PCR kit (Ambion, Life Technologies, USA). Amplification was performed in the ABI 7500 real-time PCR system thermocycler (Applied Biosystems, USA) and the following cycling conditions were used: $15 \mathrm{~min}$ at $50^{\circ} \mathrm{C}, 10 \mathrm{~min}$ at $95^{\circ} \mathrm{C}$ and 40 cycles of $8 \mathrm{~s}$ at $95^{\circ} \mathrm{C}$ and $34 \mathrm{~s}$ at $60^{\circ} \mathrm{C}$. The fluorescence reading was taken in the $60^{\circ} \mathrm{C} / 34 \mathrm{~s}$ step in each cycle and the threshold cycle $\left(\mathrm{C}_{\text {f }}\right)$ values were determined by manual adjustment. Each sample was amplified in six parallel reactions, which contained primers and probes for four different targets, detecting viruses and pathogenic bacteria, in addition to the internal control of the reaction. The positive and negative virus plasmid controls provided in the kit were included in all runs to monitor the assay performance.

Exploratory analysis of the data was performed using the SPSS software package v.17.01 (SPSS Inc, USA).

Ethics - The Research Ethical Committee of the Centre for Health Sciences of Federal University of Espírito Santo approved this study, with the registration CEP-093/07.

\section{RESULTS}

Here, we present the results of patients who met all inclusion criteria and whose samples were analysed by both methods proposed in this study. One hundred sixty-two outpatients (79 male and 83 female) were included. The study consisted of both adult $(n=46)$ and paediatric $(n=$ 116) individuals. The paediatric age group consisted of individuals zero-19 years of age, including children and adolescents, according to the World Health Organization criteria. The median age of the entire cohort was seven years (range 1 month to 75 years). Children under nine years of age accounted for $66.7 \%(108 / 162)$ of the samples analysed. All patients had recent ( $\leq 5$ days) symptoms.

IIF detected 33 positive specimens (20.4\%). The influenza virus was the most common etiologic agent detected (23/33). Inf A and Inf B corresponded to 8\% $(13 / 162)$ and $6.2 \%(10 / 162)$ of the samples, respectively. The IIF technique detected only single infections. Eighty-eight (54.3\%) specimens were positive for one or more respiratory viruses by multiplex real-time RT-PCR
(Table I). The influenza virus (15.4\%), HRV (8\%) and RSV (7.4\%) were the viruses most frequently detected and accounted for one-third of the positive samples.

Regarding the patient age groups, the influenza viruses were more frequently identified in young adults (20-39 years of age). All viruses were identified in infants (0-2 years old), with the exception of PIV 2, PIV 4 and EV. RSV and HRV were the most prevalent. Most of the samples positive for RSV (91.6\%) and HRV (77\%) were identified in infants. No virus was identified in subjects above 40 years old (Figure).

The following bacteria were detected: Streptococcus pneumoniae $[\mathrm{n}=43(26.3 \%)]$, Staphylococcus aureus $[\mathrm{n}=20(12.3 \%)]$ and Haemophilus influenzae $[\mathrm{n}=$ $2(1.2 \%)]$; Mycoplasma pneumoniae and Chlamydophila pneumoniae were not detected (Table I).

Six specimens $(3.7 \%)$ were positive for two viruses. All co-detections occurred in children under two years of age and RSV accounted for half of those cases (Table II). No co-detections involved three or more respiratory viruses. Bacterial pathogens were identified in 27 codetection cases, with at least one infectious agent from the viral or bacterial pathogen groups (Table II).

\section{DISCUSSION}

The laboratory diagnosis of viral respiratory tract infections is usually accomplished through conventional techniques, such as culture or antigen detection tests, with limitations related to the delay of the results and the availability of monoclonal antibodies for newly identified viruses (Mahony et al. 2007). Multiplex PCR assays have become an important tool for the identification of etiologic agents in ARIs (Dabisch-Ruthe et al. 2012, Sanghavi et al. 2012). As described recently, the com-

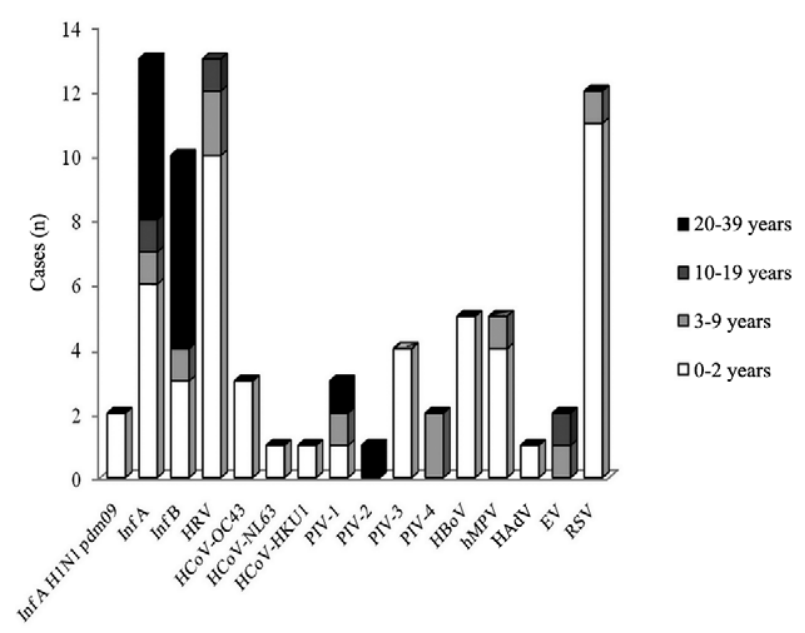

Distribution of respiratory virus of outpatients by age groups identified in nasopharyngeal aspirate by multiplex real-time reverse transcriptase polymerase chain reaction and indirect immunofluorescence assay in Vitória, Southeast Brazil, 2007-2009. EV: enterovirus; HAdV: human adenovirus; HBoV: human bocavirus; HCoV: human coronavirus; hMPV: human metapneumovirus; HRV: human rhinovirus; Inf: influenza virus; Inf A H1N1 pdm09: Inf A H1N1 pandemic; PIV: parainfluenza virus; RSV: respiratory syncytial virus. 
TABLE I

Respiratory pathogens identified in nasopharyngeal aspirate of outpatients by multiplex real-time reverse transcriptase polymerase chain reaction (RT-PCR) and indirect immunofluorescence (IIF) assay in Vitória, Southeast Brazil, 2007-2009

\begin{tabular}{|c|c|c|c|c|}
\hline \multirow[b]{3}{*}{ Pathogens } & \multicolumn{4}{|c|}{ Positive by } \\
\hline & Paediatric $^{a}$ & Adult & Paediatric $^{a}$ & Adult \\
\hline & \multicolumn{2}{|c|}{$\begin{array}{c}\text { IIF } \\
\mathrm{n}(\%)\end{array}$} & \multicolumn{2}{|c|}{$\begin{array}{c}\text { Multiplex real-time RT-PCR } \\
\text { n (\%) }\end{array}$} \\
\hline \multicolumn{5}{|l|}{ Viral agents } \\
\hline Inf A H1N1 pdm09 & NA & NA & $2(1.2)$ & $0(0)$ \\
\hline $\operatorname{Inf} A$ & $5(3.2)$ & $8(4.8)$ & $5(3.2)$ & $8(4.8)$ \\
\hline Inf B & $5(3.1)$ & $5(3.1)$ & $5(3.1)$ & $5(3.1)$ \\
\hline HRV & NA & NA & $12(6.75)$ & $2(1.25)$ \\
\hline HCoV-229E & NA & NA & $0(0)$ & $0(0)$ \\
\hline $\mathrm{HCoV}-\mathrm{OC} 43$ & NA & NA & $2(1.2)$ & $1(0.6)$ \\
\hline HCoV-NL63 & NA & NA & $1(0.6)$ & $0(0)$ \\
\hline HCoV-HKU1 & NA & NA & $1(0.6)$ & $0(0)$ \\
\hline PIV-1 & $2(1.2)$ & $0(0)$ & $2(1.2)$ & $1(0.6)$ \\
\hline PIV-2 & $0(0)$ & $0(0)$ & $0(0)$ & $1(0.6)$ \\
\hline PIV-3 & $2(1.2)$ & $0(0)$ & $3(1.8)$ & $1(0.6)$ \\
\hline PIV-4 & NA & NA & $2(1.2)$ & $0(0)$ \\
\hline $\mathrm{HBoV}$ & NA & NA & $5(3.1)$ & $0(0)$ \\
\hline hMPV A/B & NA & NA & $4(2.5)$ & $1(0.6)$ \\
\hline HAdV & $0(0)$ & $0(0)$ & $1(0.6)$ & $0(0)$ \\
\hline EV & NA & NA & $2(1.2)$ & $0(0)$ \\
\hline $\mathrm{HPeV}$ & NA & NA & $0(0)$ & $0(0)$ \\
\hline RSV A/B & $6(3.7)$ & $0(0)$ & $12(7.4)$ & $0(0)$ \\
\hline \multicolumn{5}{|l|}{ Bacterial agents } \\
\hline Streptococcus pneumoniae & NA & NA & $40(24.5)$ & $3(1.8)$ \\
\hline Staphylococcus aureus & NA & NA & $16(9.9)$ & $4(2.4)$ \\
\hline Haemophilus influenzae & NA & NA & $2(1.2)$ & $0(0)$ \\
\hline Chlamydophila pneumoniae & NA & NA & $0(0)$ & $0(0)$ \\
\hline Mycoplasma pneumoniae & NA & NA & $0(0)$ & $0(0)$ \\
\hline
\end{tabular}

a: zero-19 years of age; EV: enterovirus; HAdV: human adenovirus; HBoV: human bocavirus; HCoV: human coronavirus; hMPV: human metapneumovirus; HPeV: human parechovirus; HRV: human rhinovirus; Inf: influenza virus; Inf A H1N1 pdm09: Inf A H1N1 pandemic; NA: not applicable; PIV: parainfluenza virus; RSV: respiratory syncytial virus.

mercial multiplex FTDRP real-time RT-PCR assay has enhanced the diagnosis of ARIs, with simultaneous detection of 16 respiratory viruses (Sakthivel et al. 2012). The new version of the FTDRP assay used in this study has expanded the capacity for detecting pathogens (18 viral and 5 bacterial species) and has thus increased the diagnostic potential of the test.

More than half $(66.7 \%)$ of the study population were children under nine years of age, which is representative of the occurrence of ARIs in the community. The paediatric age group is the main group affected by ARIs, which occur three-eight times a year in infants and young children, with incidences varying inversely to age (Bryce et al. 2005, Bezerra et al. 2011).

The viral agents most frequently identified were influenza viruses, including Inf A (H1N1) pdm09 (2 cases in patients with uncomplicated respiratory infection), followed by HRV and RSV. Since April 2009, the Inf A (H1N1) pdm09 virus has spread worldwide. Given that the country's health authorities only declared sustainable transmission of the new influenza virus in Brazil (MS 2009) on 16 July 2009, we assume that the observed low frequency of Inf A (H1N1) pdm09 is related to the endpoint of the present study, in August 2009.

The frequency of influenza virus infection observed in this study (15.4\%) is consistent with previously published rates varying from $2-26 \%$ of ARI cases in studies in South, Southeast and Northeast Brazil (Arruda et al. 1991, Bellei et al. 2008). Although wide inter-regional variations should be expected in a large country with different climates, the variation may also be due to study design differences. All viruses screened in this study were 
TABLE II

Co-detection of all respiratory pathogens identified in nasopharyngeal aspirate of outpatients by multiplex real-time reverse transcriptase polymerase chain reaction (RT-PCR) in Vitória, Southeast Brazil, 2007-2009

\begin{tabular}{lc}
\hline Viruses and/or bacteria & $\mathrm{n}(\%)$ \\
\hline RSV A/B + Inf A & $1(0.6)$ \\
RSV A/B + HCoV-NL63 & $1(0.6)$ \\
RSV A/B + HBoV & $1(0.6)$ \\
RSV A/B + Streptococcus pneumoniae & $1(0.6)$ \\
RSV A/B + S. pneumoniae + Staphylococcus aureus & $1(0.6)$ \\
RSV A/B + Inf A + S. pneumoniae + Haemophilus influenzae & $1(0.6)$ \\
HRV + PIV-3 & $1(0.6)$ \\
HRV + HBoV & $1(0.6)$ \\
HRV + S. pneumoniae & $4(2.5)$ \\
HRV + PIV-3 + S. pneumoniae & $1(0.6)$ \\
hMPV A/B + HCoV-OC43 & $1(0.6)$ \\
hMPV A/B + S. pneumoniae & $2(1.25)$ \\
hMPV A/B + S. pneumoniae + S. aureus & $1(0.6)$ \\
Inf A + S. aureus & $1(0.6)$ \\
Inf A + Inf A H1N1 pdm09 + S. aureus & $2(1.25)$ \\
Inf B + S. pneumoniae & $3(1.8)$ \\
Inf B + S. aureus & $1(0.6)$ \\
PIV-1 + S. pneumoniae & $2(1.25)$ \\
PIV-3 + S. pneumoniae & $2(1.25)$ \\
PIV-4 + S. pneumoniae & $1(0.6)$ \\
PIV-4 + H. influenzae & $1(0.6)$ \\
EV + S. pneumoniae & $1(0.6)$ \\
S. pneumoniae + S. aureus & $2(1.25)$ \\
\hline
\end{tabular}

EV: enterovirus; HBoV: human bocavirus; HCoV: human coronavirus; hMPV: human metapneumovirus; HRV: human rhinovirus; Inf: influenza virus; Inf A H1N1 pdm09: Inf A H1N1 pandemic; PIV: parainfluenza virus; RSV: respiratory syncytial virus.

more frequently identified in children, with the exception of influenza viruses, which were more often identified in adults $(8.6 \%)$ than in paediatric patients $(6.8 \%)$, as has been documented in a recent study (Pelat et al. 2013).

The second most prevalent viral agent was HRV (8\%): $77 \%$ of HRV-positive samples were identified in children under two years of age. This finding highlights the importance of this virus in infants, as HRV has been associated with recurrent respiratory illnesses and wheezing in this age group (Jartti et al. 2008). Other picornaviruses may be etiologic agents of ARIs; however, HRV is the main virus detected in cases of the common cold (Pitkäranta \& Hayden 1998). In a multicentre study, rhinoviruses were often associated with ARIs, even during the peak influenza season (Caruso et al. 2007). In Brazil, according to Arruda et al. (1991), HRV was the most frequent viral agent $(46 \%)$ detected in children with ARIs.

The frequency of RSV detection (7.4\%) was similar to that detected by Raboni et al. (2011), who reported a rate of $8 \%$ using multiplex RT-PCR for 14 respiratory viruses. Eleven (91.6\%) of the RSV cases in this study were detected in children under two years of age. RSV antibodies are found in virtually all adults and children older than three years of age. At one year of age, 25-
$50 \%$ of children have antibodies against RSV, demonstrating the high frequency of this infection at a young age (Domachowske \& Rosenberg 1999). In keeping with this observation, the frequency of RSV in the youngest age group in our study was the highest among all ages $(91.6 \%)$. However, the study was conducted with patients of widely variable ages: only two-thirds were children under nine and only $40 \%$ of the studied patients were children under two years of age. Hence, it is reasonable to consider that the observed relatively low frequency of RSV could be attributed, at least in part, to the underrepresentation of patients of very young ages.

The frequency of $\mathrm{HBoV}$ was $3.1 \%$ and all cases occurred in children under two years of age. This virus has been frequently identified in this age group, although its pathogenicity is not yet well defined (Longtin et al. 2008, Lüsebrink et al. 2009). Recently, a higher HBoV-positive rate has been documented in inpatients when compared with outpatients or patients attended in emergency departments, suggesting a significant role of this virus in the pathogenesis of ARIs (Xu et al. 2012).

The use of multiplex real-time RT-PCR increased the overall viral detection rate to $54.3 \%$, compared to $20.4 \%$ in the IFF test. This difference is likely due to the 
high sensitivity of virus genome detection (Lassaunière et al. 2010). In addition, $47.9 \%$ of the samples that were positive by multiplex real-time RT-PCR corresponded to respiratory viruses that were not tested for using the IIF kit, such as HRV, HCoV, hMPV, HBoV and EV. This finding confirms the importance of using panels that identify multiple viral agents causing ARIs.

In this study, there were six cases $(3.7 \%)$ of viral codetection, most commonly with RSV and HRV. The real clinical significance of these infections has not yet been fully elucidated. In a recent Brazilian study, co-detection between RSV and HRV increased both the length of hospitalisation and the time of supplemental oxygen use in children with lower respiratory tract infections, suggesting the possibility of co-infection among these viruses and not only co-detection (da Silva et al. 2013). All cases of co-detection observed in our study occurred in children under two years old, the age group in which ARI cases are more frequent. It is quite possible that residual nucleic acids from sequential viral infections are detected simultaneously by multiplex PCR with high sensitivity (Lesley 2012). Additionally, lymphoid tissues may serve as a reservoir of respiratory viruses in asymptomatic individuals and may be involved in the transmission of these viruses to the community (Proenca-Modena et al. 2012).

The likelihood of a pathogen to be the aetiologic agent of a given infection could be related to the $C_{t}$ value in the real-time PCR reaction. Lower $\mathrm{C}_{\mathrm{t}}$ values suggest higher loads of the pathogen detected, which may in turn suggest aetiology (Brittain-Long et al. 2008). In this study, the $\mathrm{C}_{\mathrm{t}}$ values were well below the cut-off value for positivity proposed by the manufacturer.

The overall bacterial detection rate was $40 \%$ and $S$. pneumoniae was the most frequent agent identified. Despite the vaccination program, S. pneumoniae is one of the main causes of pneumonia mortality (responsible for at least $18 \%$ of severe episodes and $33 \%$ of deaths worldwide) (Walker et al. 2013). Bacteria were co-detected with viruses in 25 specimens. The potentially pathogenic bacteria detected in this study could reflect transient microbiota or a nasopharyngeal flora and may not be associated with ARIs; however, it is well known that viral infections within the respiratory tract predispose the individual to bacterial infections, notably through the disruption of the respiratory mucosal epithelium (Bakaletz 1995). Atypical bacteria (M. pneumoniae and C. pneumonia) were not detected in the studied population.

In Brazil and other countries, the respiratory virus surveillance programs use conventional diagnostic techniques, such as IIF. A large proportion of biological samples remain negative, even with clinical evidence of respiratory infection. A molecular technique such as real-time PCR can be an important tool, increasing the detection capacity of a large number of respiratory pathogens. Moreover, this type of technique can contribute to the correct indication of antiviral medications, can avoid unnecessary use of antibiotics and can promote the adoption of appropriate hospital-control measures.

The findings of this study show that the application of PCR assays in a real-time multiplex format for respiratory pathogens considerably increases the pathogen detection rate when compared to conventional methods, highlighting the role of influenza virus in ARI cases in patients of all ages, in addition to reporting the circulation of recently described respiratory viruses (Inf A H1N1 pdm09, hMPV, HBoV, HCoV-NL63 and HCoV-HKU1).

\section{REFERENCES}

Allander T, Tammi MT, Eriksson M, Bjerkner A, Tiveljung-Lindell A, Andersson B 2005. Cloning of a human parvovirus by molecular screening of respiratory tract samples. Proc Natl Acad Sci 102: 12891-12896.

Arruda E, Cintra OAL, Hayden FG 2006. Respiratory tract viral infections. In RL Guerrant, Tropical infectious diseases - Principles, pathogens and practice, Vol. I, Churchill Livingstone Elsevier, Philadelphia, p. 637-659.

Arruda E, Hayden FG, McAuliffe JF, de Sousa MA, Mota SB, McAuliffe MI, Geist FC, Carvalho EP, Fernandes MC, Guerrant RL 1991. Acute respiratory viral infections in ambulatory children of urban Northeast Brazil. J Infect Dis 164: 252-258.

Bakaletz LO 1995. Viral potentiation of bacterial superinfection of the respiratory tract. Trends Microbiol 3: 110-114.

Barenfanger J, Drake C, Leon N, Mueller T, Troutt T 2000. Clinical and financial benefits of rapid detection of respiratory viruses: an outcomes study. J Clin Microbiol 38: 2824-2828.

Bellei N, Carraro E, Perosa A, Watanabe A, Arruda E, Granato C 2008. Acute respiratory infection and influenza-like illness viral etiologies in Brazilian adults. J Med Virol 80: 1824-1827.

Bezerra PG, Britto MC, Correia JB, Duarte MC, Fonceca AM, Rose K, Hopkins MJ, Cuevas LE, McNamara OS 2011. Viral and atypical bacterial detection in acute respiratory infection in children under five years. PLOS ONE 6: e18928.

Brittain-Long R, Nord S, Olofsson S, Westin J, Anderson LM, Lindh M 2008. Multiplex real-time PCR for detection of respiratory tract infections. J Clin Virol 41: 53-56.

Brittain-Long R, Westin J, Olofsson S, Lindh M, Andersson LM 2010. Prospective evaluation of a novel multiplex real-time PCR assay for detection of fifteen respiratory pathogens - duration of symptoms significantly affects detection rate. J Clin Virol 47: 263-267.

Bryce J, Boschi-Pinto C, Shibuya K, Black RE 2005. WHO estimates of the causes of death in children. WHO Child Health Epidemiology Reference Group. Lancet 365: 1147-1152.

Caruso TJ, Prober CG, Gwaltney Jr JM 2007. Treatment of naturally acquired common colds with zinc: a structures review. Clin Infect Dis 45: 569-574.

da Silva ER, Pitrez MC, Arruda E, Mattiello R, Sarria EE, de Paula FE, Proença-Modena JL, Delcaro LS, Cintra O, Jones MH, Ribeiro JD, Stein RT 2013. Severe lower respiratory tract infection in infants and toddlers from a non-affluent population: viral etiology and co-detection as risk factors. BMC Infect Dis 13: 41.

Dabisch-Ruthe M, Vollmer T, Adams O, Knabbe C, Dreier J 2012. Comparison of three multiplex PCR assays for the detection of respiratory viral infections: evaluation of XTAG respiratory virus panel fast assay, RespiFinder 19 assay and RespiFinder SMART 22 assay. BMC Infect Dis 12: 163.

Domachowske JB, Rosenberg HF 1999. Respiratory syncytial virus infection: immune response, immunopathogenesis and treatment. Clin Microbiol Rev 12: 298-309.

Gunson RN, Collins TC, Carman WF 2005. Real-time RT-PCR detection of 12 respiratory viral infections in four triplex reactions. J Clin Virol 33: 341-344. 
Jartti T, Lee WM, Pappas T, Evans M, Lemanske Jr RF, Gern JE 2008. Serial viral infections in infants with recurrent respiratory illnesses. Eur Respir J 32: 314-320.

Lassaunière R, Kresfelder T, Venter M 2010. A novel multiplex realtime RT-PCR assay with FRET hybridization probes for the detection and quantitation of 13 respiratory viruses. $J$ Virol Methods 165: 254-260.

Lesley P 2012. The complication of coinfection. Yale J Biol Med 85: 127-132.

Longtin J, Bastien M, Gilca R, Leblanc E, de Serres G, Bergeron MG, Boivin G 2008. Human bocavirus infections in hospitalized children and adults. Emerg Infect Dis 14: 217-221.

Lüsebrink J, Wittleben F, Schildgen V, Schildgen O 2009. Human bocavirus - insights into a newly identified respiratory virus. $\mathrm{Vi}$ ruses 1: 3-12.

Mahony J, Chong S, Merante F, Yaghoubian S, Sinha T, Lisle C, Janeczko R 2007. Development of a respiratory virus panel test for detection of twenty human respiratory viruses by use of multiplex PCR and a fluid microbead-based assay. J Clin Microbiol 45: 2965-2970.

Mahony JB 2008. Detection of respiratory viruses by molecular methods. Clin Microbiol Rev 21: 716-747.

MS - Ministério da Saúde Brasil 2009. Informe epidemiológico: influenza pandêmica (H1N1). Available from: portal.saude.gov.br/ portal/arquivos/pdf/boletim_influenza_se_47.pdf.

Osterhaus AD 2008. New respiratory viruses of humans. Pediatr Infect Dis J 27: 71-74.

Pelat C, Lasserre A, Xavier A, Turbelin C, Blanchon T, Hanslik T 2013. Hospitalization of influenza-like illness patients recommended by general practitioners in France between 1997 and 2010. Influenza Other Respir Viruses 7: 74-84.

Pitkäranta A, Hayden FG 1998. Rhinoviruses: important respiratory pathogens. Ann Med 30: 529-537.

Proenca-Modena JL, Pereira VFC, Jacob MG, Buzatto GP, Saturno TH, Lopes L, Souza JM, Escremim PF, Silva ML, Carenzi LR,
Tamashiro E, Arruda E, Anselmo LWT 2012. High rates of detection of respiratory viruses in tonsillar tissues from children with chronic adenotonsillar disease. PLoS ONE 7: e42136.

Raboni SM, Stella V, Cruz CR, França JB, Moreira S, Gonçalves L, Nogueira MB, Vidal LR, Almeida SM, Debur MC, Carraro Jr H, dos Santos CN 2011. Laboratory diagnosis, epidemiology and clinical outcomes of pandemic influenza A and community respiratory viral infections in southern Brazil. J Clin Microbiol 49: 1287-1293.

Sakthivel SK, Whitaker B, Lu X, Oliveira DB, Stockman LJ, Kamili S, Oberste MS, Erdman DD 2012. Comparison of fast-track diagnostics respiratory pathogens multiplex real-time RT-PCR assay with in-house singleplex assay for comprehensive detection of human respiratory viruses. $J$ Virol Methods 185: 259-266.

Sanghavi SK, Bullotta A, Husain S, Rinaldo CR 2012. Clinical evaluation of multiplex real-time PCR panels for rapid detection of respiratory viral infections. J Med Virol 84: 162-169.

van den Hoogen BG, de Jong JC, Groen J, Kuiken T, de Groot R, Fouchier RA, Osterhaus AD 2001. A newly discovered human pneumovirus isolated from young children with respiratory tract disease. Nat Med 7: 719-724.

van der Hoek L, Pyrc K, Jebbink MF, Vermeulen-Oost W, Berkhout RJ, Wolthers KC, Wertheim-van Dillen PM, Kaandorp J, Spaargaren J, Berkhout B 2004. Identification of a new human coronavirus. Nat Med 10: 368-373.

Walker CL, Rudan I, Liu L, Nair H, Theodoratou E, O’Brien KL, Campbell H, Black RE 2013. Global burden of childhood pneumonia and diarrhoea. Lancet 381: 1405-1416.

Woo PC, Lau SK, Chu CM, Chan KH, Tsoi HW, Huang Y, Wong BH, Poon RW, Cai JJ, Luk WK, Poon LL, Wong SS, Guan Y, Peiris JS, Yuen KY 2005. Characterization and complete genome sequence of a novel coronavirus, coronavirus HKU1, from patients with pneumonia. J Virol 79: 884-895.

Xu L, He X, Zhang D-m, Feng F-s, Wang Zl 2012. Surveillance and genome analysis of human bocavirus in patients with respiratory infection in Guangzhou, China. PLoS ONE 7: e44876. 